\title{
Research on the Efficiency of Minesweeping Operation for Magnetic Minesweeping Gear
}

\author{
Huidong Lü, a Xinqin Chen ${ }^{1, b}$, Chang Shu', Wei Zhang ${ }^{1}$, Xuanmin $\mathrm{Li}^{2}$, \\ Longteng $\mathrm{Li}^{1}$, Chengwen Zhu ${ }^{1}$
}

${ }^{1}$ Dept. of Command and control, Wuhan Ordnance Officer School, Wuhan, Hubei, 430075, China

${ }^{2}$ Dept. of Computer, Wuhan Bridge College, Wuhan, Hubei, 430052, China

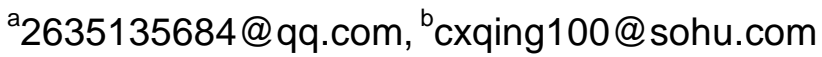

Keywords: mine fuze magnetic minesweeping gear isomagnetic line minesweeping width Abstract. In minesweeping operations, it is difficult to get optimum sweeping width due to complicated magnetic field distribution. Therefore the main work in this dissertation is to find a method to analyze the characteristics of magnetic field and key factors which can affect minesweeping width. First, the distribution model of magnetic field is given according to different magnet configurations. Second, the isomagnetic lines are drawn and disscussed for different components. Finally, the optimum sweeping width is obtained by the characteristics of isomagnetic lines. The study result shows that different magnet configurations will have a great impact on the effect of mine sweeping. In addition, the choice of different components will also determine the effect of mine sweeping.

\section{Introduction}

It is known that when the magnetic field of the magnetic minesweeping gear has reached the point which is called threshold of mine, the mine can be wiped off. It is related to magnetic field strength that whether the mine fuze is triggered or not[1-2]. However, for a minefield, the magnetic field of the magnetic minesweeping gear is not uniform, its polarity and magnitude are not the same in different locations, and even in some locations there may be dead zones [3-7]. Sometimes, although the magnetic field is very large, the mine sweeping width may be small due to the influence of the dead zone. For the multi magnet, the final formation of the magnetic field may be enhanced or weakened owing to the interactions with the parts of magnetic field, and it will result in a corresponding change in the minesweeping width. Increasing the minesweeping width, reducing the dead zone and improving the efficiency of mine clearance are important indicators to measure the efficiency of minesweeping operations, as well as the main basis for the development of minesweeping plan. In this paper, the magnetic field is studied based on three possible arrangements of magnets: longitudinal, transverse, vertical. The effect of mine sweeping is analyzed according to distribution of isomagnetic line under different circumstances.

\section{The magnetic field ditribution models}

Combined with actual situation of magnetic minesweeping gear, this paper adopts magnetic dipole model based on the magnetization of single direction. According to different deployment, the models can be described as follows:

magnetic field distribution of longitudinal magnetic dipole

As shown in Fig. 1, the magnetic field generated by longitudinal magnetic dipole located at space point $P(x, y, z)$ is: 


$$
\begin{aligned}
& \left\{\begin{array}{l}
H_{x i}=\frac{M_{x i}}{4 \pi}\left(\frac{3\left(x-x_{i}\right)^{2}}{r_{i}^{5}}-\frac{1}{r_{i}^{3}}\right) \\
H_{y i}=\frac{M_{x i}}{4 \pi}\left(\frac{3\left(x-x_{i}\right) y}{r_{i}^{5}}\right) \\
H_{z i}=\frac{M_{x i}}{4 \pi}\left(\frac{3\left(x-x_{i}\right) z}{r_{i}^{5}}\right)
\end{array}\right. \\
& \text { Where: } r_{i}=\sqrt{\left(x-x_{i}\right)^{2}+y^{2}+z^{2}} \\
& \longrightarrow
\end{aligned}
$$

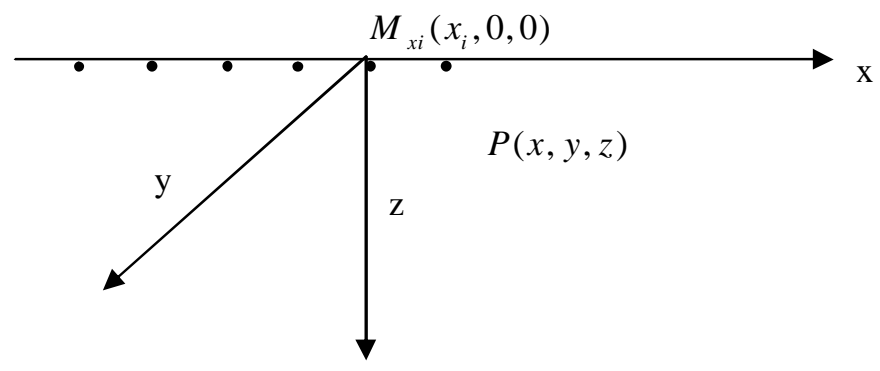

Fig. 1 Coordinates and illustrative chart of magnetic dipole

When linear array pass through from the measuring point, the coordinates $\mathrm{y}, \mathrm{z}$ remain unchanged while $\mathrm{x}$ changing. The magnetic field $T$ generated at the measuring point will take the maximum value where $x=x_{i}$. The maximum value of magnetic field can be obtained as follows:

$$
T_{\max }=\max \left\{\sqrt{H_{x i}^{2}+H_{y i}^{2}+H_{z i}^{2}}\right\}=\frac{M_{x i}}{4 \pi \sqrt{\left(y^{2}+z^{2}\right)^{3}}}
$$

\section{magnetic field distribution of transverse magnetic dipole}

For transverse magnetic dipole, the distribution of magnetic field is similar to that of longitudinal magnetic dipole. The magnetic field of it can be stated as follows:

$$
\left\{\begin{array}{l}
H_{x i}=\frac{M_{y i}}{4 \pi}\left(\frac{3\left(x-x_{i}\right) y}{r_{i}^{5}}\right) \\
H_{y i}=\frac{M_{y i}}{4 \pi}\left(\frac{3\left(x-x_{i}\right)^{2}}{r_{i}^{5}}-\frac{1}{r_{i}^{3}}\right) \\
H_{z i}=\frac{3 M_{y i}}{4 \pi} \cdot \frac{y z}{r_{i}^{5}}
\end{array}\right.
$$

Where: $r_{i}=\sqrt{\left(x-x_{i}\right)^{2}+y^{2}+z^{2}}$

In the same way, the maximum value of magnetic field: $T_{\max }=\frac{M_{x i}}{4 \pi \sqrt{\left(y^{2}+z^{2}\right)^{3}}}$

\section{magnetic field distribution of vertical magnetic dipole}

In Fig. 1, if the longitudinal magnetic dipole is replaced by a vertical magnetic dipole, the magnetic field at the point $\mathrm{P}(\mathrm{x}, \mathrm{y}, \mathrm{z})$ is:

$$
\left\{\begin{array}{l}
H_{x i}=\frac{3 M_{z i}}{4 \pi}\left(\frac{z\left(x-x_{i}\right)}{r_{i}^{5}}\right) \\
H_{y i}=\frac{3 M_{z i}}{4 \pi} \cdot \frac{y z}{r_{i}^{5}} \\
H_{z i}=\frac{M_{z i}}{4 \pi}\left(\frac{3 z^{2}}{r_{i}^{5}}-\frac{1}{r_{i}^{3}}\right)
\end{array}\right.
$$


The magnetic field $T$ will take the maximum value where $x=x_{i}$ :

$$
T_{\max }=\max \left\{\sqrt{H_{x i}^{2}+H_{y i}^{2}+H_{z i}^{2}}\right\}=\frac{M_{z i}}{4 \pi \sqrt{\left(y^{2}+z^{2}\right)^{3}}} \cdot \sqrt{1+\frac{3 z^{2}}{y^{2}+z^{2}}}
$$

From type (2) and (5), it can be seen that magnetic field generated by the vertical magnetic dipole is larger than that of the longitudinal magnetic dipole. When the transverse coordinate $y$ and water depth $z$ is equal, the numerical value of difference is about 1.6 times.

\section{Analysis of Isomagnetic Line}

\section{longitudinal magnet}

Given the following parameters: minesweeping speed $v=12 \mathrm{kn}$, sea depth $h=10 \mathrm{~m}$, mine sensitivity $H_{s}=3 m O e$. Supose the shortest time required for mine fuze reliable action is $2 \mathrm{~s}$, introducing a single longitudinal magnet whose magnetic moment is $99 \times 10^{3} \mathrm{~A}^{\mathrm{m}} \mathrm{m}^{2}$ as the subject, we can obtain the distribution of isomagnetic line as shown in Fig. 2 and minesweeping width: 58/0m, $62 / 25 \mathrm{~m}, 38 / 1 \mathrm{~m}$.
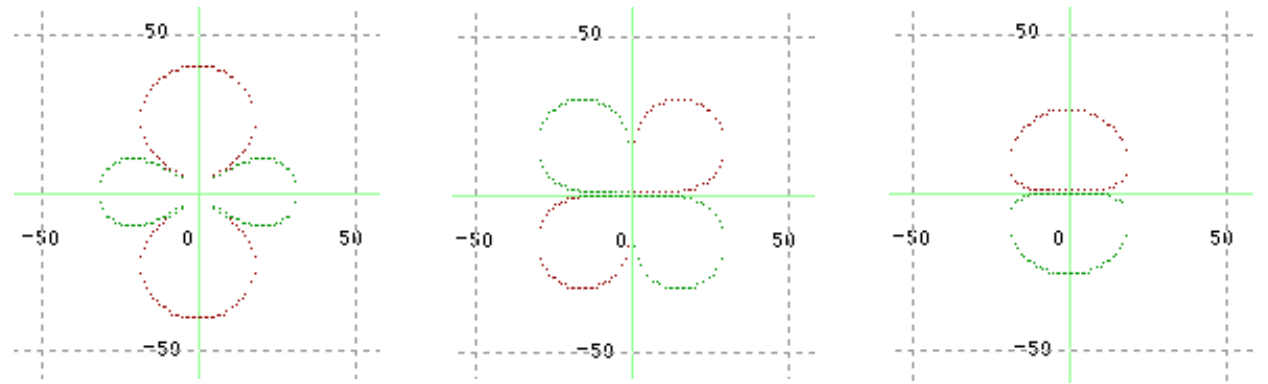

Fig. 2 The isomagnetic line of single longitudinal magnet $\left(H_{x}, H_{y}, H_{z}\right)$

In Fig. 2, the component of the magnetic field $H_{x}$ has 3 magnetic field regions: front, middle, rear. The front and rear have opposite polarities compared with the middle, and the front can extend far forward without dead region. We use the front region to sweep mine due to its good reliability. For the component of the magnetic field $H_{y}$, there are 4 magnetic field regions that can bring a larger minesweeping width along with a small dead zone along the center of the minesweeping gear. Unlike other two components, the component $H_{z}$ has 2 magnetic field regions which have the opposite polarities, and its minesweeping effect is poor.

\section{transverse magnet}

The magnet is placed horizontally and the other parameters of it are not changed. The isomagnetic line of component $\left(H_{x}, H_{y}\right.$ and $H_{z}$ ) can be obtained as shown in Fig. 3, then the minesweeping width is $58 / 0 \mathrm{~m}, 78 / 7 \mathrm{~m}$ and $50 / 8 \mathrm{~m}$.
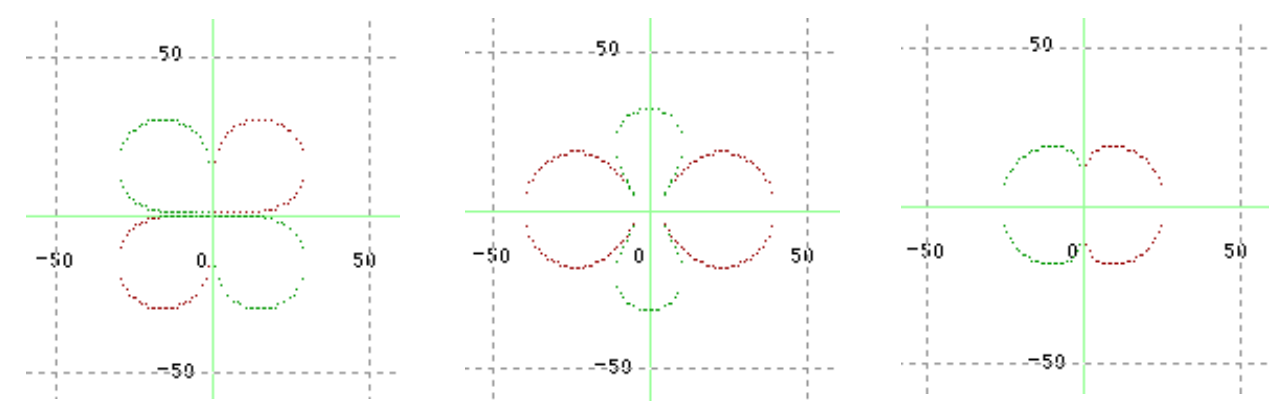

Fig. 3 The isomagnetic line of one transverse magnet $\left(H_{x}, H_{y}, H_{z}\right)$

Further analysis reveals that the magnetic components of $H_{y}$ and $H_{z}$ are enhanced obviously, and 
the minesweeping width is greatly increased, but the magnetic of $H_{x}$ component is weakened.

\section{vertical magnet}

Keeping other parameters constant, we put the magnet vertically. The isomagnetic line of component $\left(H_{x}, H_{y}\right.$ and $\left.H_{z}\right)$ can be obtained as shown in Fig. 4 with the corresponding width: $38 / 1 \mathrm{~m}$, $50 / 8 \mathrm{~m}$ and $60 / 3 \mathrm{~m}$.

At this time, the component $H_{z}$ is improved, but the other two components of the magnetic field are relatively weakened.
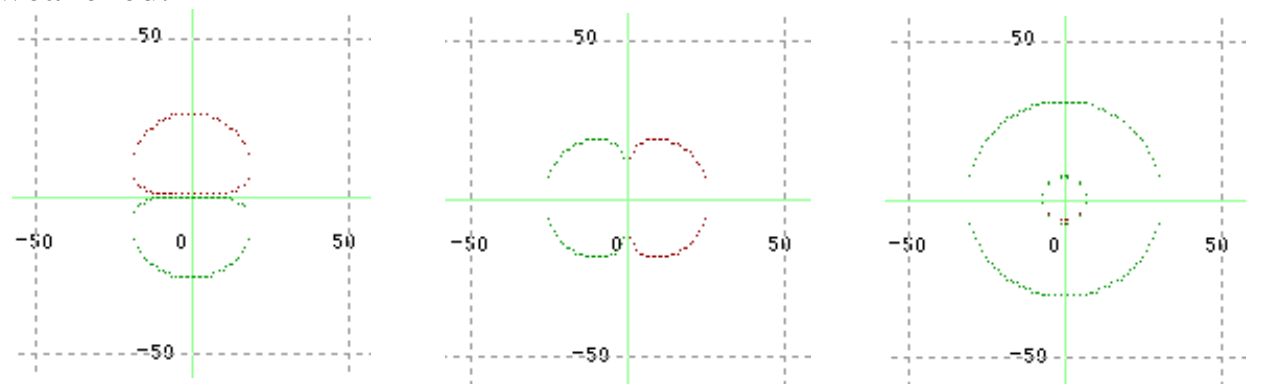

Fig. 4 The isomagnetic line of one vertical magnet $\left(H_{x}, H_{y}, H_{z}\right)$

\section{Discussions and Conclusions}

The calculation results of the three configurations show that the minesweeping effect of transverse magnet is the best, followed by that of longitudinal magnet and lastly by that of vertical magnet. In addition, the magnetic field of each part is influenced by the superposition, it may be enhanced or weakened, resulting in the corresponding changes in the minesweeping width.

If using component $H_{x}$ to take minesweeping operation, we can put magnets longitudinal cloth, and make magnetic field polarity opposite to the adjacent magnet, then get lager minesweeping width. For the other two components $H_{y}$ and $H_{z}$, in order to obtain larger minesweeping width, the former requires a transverse arrangement of the magnet with the same polarity to adjacent one, and the latter requires a longitudinal arrangement of the magnet with the same polarity to adjacent one.

\section{References}

[1] Xinqin Chen, Wei Zhang, Longteng Li, etc. Research on Modeling Method of Ship Magnetic Field Based on Magnetic Minesweeping Gear. ETEEEM (2014):363-366

[2] Fu Jin-zhu.2004. Goal Setting Minesweeping System. Mine Warfare2 \& Ship Self-defence . (2) : 9-14.

[3] Liu Zhong-le, Zhou Shi-xi, etc.1995.Research on Control Technology of High Simulation Magnetic Electromagnetic Minesweeping Gear simulation [D]. Wuhan: Naval University of Engineering.

[4] Xinqin Chen.2009.Study on Pivotal Techniques of Mine Sweeping Based on Magnetic Fuse Mine [D]. Wuhan: Naval University of Engineering.

[5] Lin Chun-sheng.2003.The New Technology Lectures of Mine fuze[M]. Wuhan: Naval University of Engineering.

[6] Lin Chun-sheng.2006. The Development of Ship Magnetic Field Modeling and Verification of Software [R] . Wuhan: Naval University of Engineering.

[7] Lin Chun-sheng, Gong Sheng-guang. 2007. Physical field of warship [M]. Beijing: Weapon Industry Press. 